\section{Danger takes its Toll}

The nematode Caenorhabditis elegans expresses a single Toll-like receptor, TOL-1, that is involved in the avoidance of pathogens via an unknown mechanism. In Current Biology, Brandt and Ringstad show that TOL-1 promotes the development of specific sensory neurons. Expression of TOL-1 by BAG neurons is required for productive signaling via MAPK kinases and mediates their chemosensing function. The authors find that BAG neurons detect $\mathrm{CO}_{2}$, which, along with other unknown odorants, is interpreted as a signature specific to live pathogenic microbes; this leads to avoidance behavior by the nematode. The absence of TOL-1 on BAG neurons leads the nematodes to linger on an otherwise hazardous lawn of microbes. These findings directly link the nematode Toll-like receptor to a defensive behavioral response. ZF Curr. Biol. (13 August 2015) doi:doi.org/10.1016/ j.cub.2015.07.037

\section{Guiding nerve regeneration}

The regeneration of fully cut peripheral nerves requires the Schwann cell-guided regrowth of axons across bridges formed by a mixture of cells of the immune system and connective-tissue cells. In Cell, Lloyd and colleagues show that macrophages specifically sense hypoxia within the bridge environment and secrete VEGF-A to induce polarized vascularization of the bridge. The bridge is fully vascularized before the migration of Schwann cells, which closely adhere to the endothelial cell scaffold and use it as a guiding path for migration. Endothelial cells migrate in response to VEGF-A, but Schwann cells do not. Deletion of VEGF-A specifically in macrophages diminishes vascularization of the bridge and confines Schwann cells to the nerve stumps, while ectopic VEGF-A signals from the muscle mass misdirect the blood vessels, the Schwann cell cords and the axons. Thus, macrophages sense the conditions in the injured nerve and initiate the regeneration process. Cell (13 August 2015) doi:10.1016/j.cell.2015.07.021

\section{Unifying principles}

Jawless fish such as lampreys and hagfish have a humoral immune system that shows close functional convergence with the B cells and antibodies of vertebrates but is structurally completely unrelated. In eLife, Herrin and colleagues compare the reponses of mouse immunoglobulin-based antibodies and a lamprey leucine-rich repeat-based antibody, VLR-B, to influenza virus. Immunization of lampreys generates highly specific VLR-B antibodies that, much like those in the mouse, are directed almost exclusively against the globular head of influenza virus hemagglutinin (HA). HA-specific lamprey antiserum is able to block infection with influenza virus in vitro and also compete with mouse antibodies for overlapping epitopes, which suggests a very similar immunodominance hierachy for these two very unrelated species. Therefore, despite the massive structural differences between VLR-B and immunoglobulin antibodies, unifying principles of antigen recognition seem to prevail. eLife (30 July 2015) doi:10.1016/j.cell.2015.06.057

\section{Metastasis factor}

Metastasis-associated macrophages (MAMs) at metastatic sites induce angiogenesis and tumor growth, partially through production of the angiogenic factor VEGF. In the Journal of Experimental Medicine, Qian et al. show that expression of Flt1, a VEGF receptor, promotes tumor metastasis by regulating the expression of a set of pro-inflammatory genes in MAMs. Human breast tumor metastases show significant enrichment for Flt $1^{+}$ macrophages compared with their abundance in primary tumors. FIt1 signaling in macrophages is required for the formation of metastatic tumors in mice but not for the recruitment or extravasation of MAMs to the metastatic site. Ly $6 c^{+}$inflammatory monocytes, the precursors of MAMs, do not express Flt1. Among the Flt1-dependent pro-inflammatory gene signature in MAMs, expression of the gene encoding the macrophage survival factor CSF1 is required for efficient tumor metastasis. Thus, specific expression of FIt 1 on MAMs creates an inflammatory environment that allows metastatic growth after seeding.

J. Exp. Med. (10 August 2015) doi:10.1084/jem.20141555

\section{Understanding deletional CSR}

Productive immunoglobulin class-switch recombination (CSR) in activated $B$ cells joins upstream switch $\mu$-chain $\left(S_{\mu}\right)$ sequences to downstream $\mathrm{S}_{\gamma}, \mathrm{S}_{\alpha}$ or $\mathrm{S}_{\varepsilon}$ segments. In Nature, Dong et al. provide a mechanistic basis for the deletional, rather than inversional, DNA recombination in the locus encoding the immunoglobulin heavy chain (Igh). High-throughput sequencing of 'bait' and 'prey' DNA junctions reveals a significant bias for DNA deletions in switching $B$ cells. This deletional CSR bias is independent of the orientation of $S_{\mu}$ or its transcription. However, cells lacking 53BP1, a DNA-repair factor activated during CSR, lose directional recombination bias, as DNA inversions are as frequent as deletions. 53BP1 deficiency also increases the frequency of intraswitch-region 'resections', which likewise produce nonproductive CSR. Thus, 53BP1 enforces a directional bias during CSR to promote DNA deletions and thereby allow productive antibody switching from IgM to IgG, IgA or IgE. Nature (26 August 2015) doi:10.1038/nature14970

\section{Universal influenza vaccine}

The high mutation frequency of genes encoding influenza virus HA and neuraminidase prompts the need for yearly vaccination against circulating seasonal influenza virus strains. In Science, Impagliazzo et al. demonstrate the feasibility of eliciting protective broadly neutralizing antibodies to multiple heterologous strains of influenza virus. The design involves the rational introduction of specific mutations in sequence encoding the HA stem region that stabilize its trimeric conformation upon removal of the HA head region, which often masks the protective stem epitopes. The resulting 'mini-HA' has structure and glycosylation similar to that of full-length HA. These mini-HA constructs are very immunogenic in mice and monkeys and elicit broadly neutralizing antibodies that prove to be protective against heterotypic influenza virus variants. These findings offer proof of principle that an universal vaccine against influenza virus is achievable. $L A D$ Science (24 August 2015) doi:10.1126/science.aac7263 